\title{
Perception of biological motion: A stimulus set of human point-light actions
}

\author{
JAN VANRIE and KARL VERFAILLIE \\ Katholieke Universiteit Leuven, Leuven, Belgium
}

\begin{abstract}
We present a set of stimuli representing human actions under point-light conditions, as seen from different viewpoints. The set contains 22 fairly short, well-delineated, and visually "loopable" actions. For each action, we provide movie files from five different viewpoints as well as a text file with the three spatial coordinates of the point lights, allowing researchers to construct customized versions. The full set of stimuli may be downloaded from www.psychonomic.org/archive/.
\end{abstract}

An important factor in the advancement of many fields of research is the diversity of suitable stimulus material. One class of stimuli where this diversity has been rather limited is the visual representation of human actions. Indeed, stimuli that represent human actions or movements have been less available to the scientific community than stimuli of objects or animals. Moreover, the majority of published stimuli regarding human actions are generally in the form of line drawings or photographs (e.g., Fiez \& Tranel, 1997, or the object and action naming battery by Druks \& Masterson, 2000). Because these representations exclude the dynamic component of the action, they are not equally suitable for all research goals. Fortunately, a number of research groups have recently started to develop animated versions of action stimuli and methods of constructing these stimuli (see next section). In the present paper, we would like to contribute to the diversity in this domain by presenting a set of moving stimuli involving human actions under point-light conditions as seen from different viewpoints. The stimuli, which consist of both movie files and 3-D coordinate files, can be retrieved from the archival Web site of the Psychonomic Society.

\section{Point-Light Technique}

In 1973, Johansson devised a method for representing human movement through minimal visual information. In this method, the movements of a body are represented by a small number of moving bright patches indicating the major joints of a person performing some action. Despite the absence of other cues such as contour or texture, ob-

We thank Dirkjan Veeger and Steven Jax for their comments on a previous version of this manuscript. This research was supported by Concerted Research Effort Convention GOA 98/01, the Research Training Network "Perception for Recognition and Action" (RTN-2001-00107) under the direction of the European Community, and the Fund for Scientific Research of Flanders, of which J.V. is a research assistant. Correspondence should be addressed to J. Vanrie, Laboratory of Experimental Psychology, Katholieke Universiteit Leuven, Tiensestraat 102 , B-3000 Leuven, Belgium (e-mail: jan.vanrie@psy.kuleuven.ac.be). servers can quite easily identify several features of both actor and action (e.g., Ahlström, Blake, \& Ahlström, 1997; Cutting \& Kozlowski, 1977; Dittrich, 1993; Dittrich, Troscianko, Lea, \& Morgan, 1996; see Verfaillie, 2000 , for a brief overview). This point-light technique has obvious advantages, because it allows a large degree of control over the stimulus and it restricts the visual information to the bodily motion. An important drawback, however, is the complexity of constructing such "biological motion" stimuli. There are several ways to produce these stimuli (tracing body joint locations on photographs or films, direct video recording, computer animation based on artificial synthesis, or computer animation based on motion capture data; see Dekeyser, Verfaillie, \& Vanrie, 2002, for a more detailed discussion), and although the methods differ on several accounts, all are fairly time consuming or complex. An important breakthrough was the publication of an algorithm (Cutting, 1978) that allowed biological-motion researchers to generate synthetic walkers as dynamic point-light displays. Moreover, in subsequent studies, the algorithm, which was originally only for a lateral walker, was adapted to include nonlateral orientations (e.g., Verfaillie, De Troy, \& Van Rensbergen, 1994). This has made the point-light walker probably the most frequently used stimulus in biological-motion research. Of course, with this method one is still limited to a single action (walking), and given the recent surge of interest in human action perception, this has not been sufficient for tackling the relevant questions.

For this reason, a number of researchers have gone beyond the use of the Cutting algorithm and constructed their own sets of point-light actions (e.g., Ahlström et al., 1997; Dittrich, 1993; Olofsson, Nyberg, \& Nilsson, 1997). Such stimuli, however, are typically custom made for the problem at hand and not readily available. Two noteable exceptions in this case are the point-light archive established by Thomas Shipley in the Vision Lab at Temple University (available at http://astro.temple.edu/ tshipley/mocap.html) and the movement database developed by Frank Pollick in the Biological Motion and Action Understanding Labora- 
tory at the University of Glasgow (available at http://pollicklab.psy.gla.ac.uk/modules.php?name = Data). The first one consists of 79 point-light actions, 28 of which involve nonhuman animals, that were created by identifying joint locations on digital video frames. These actions are available in different movie formats or as 2-D coordinate files. The database of the Pollick lab consists of 1,500 movements. More specifically, each of 30 actors ( 15 male, 15 female) performed a limited set of actions (knocking, lifting, walking, throwing) under specific circumstances (i.e., in different emotional states). The movement patterns are (or will be) available as movie files, but also in specific file formats, allowing researchers with the appropriate software packages to manipulate the patterns.

\section{Goal}

Dekeyser et al. (2002) have described a method for creating biological-motion stimuli on the basis of motion capture data and animation software. With this method, we created a number of point-light actions, which have been used in a variety of experiments both in our laboratory (e.g., Vanrie, Dekeyser, \& Verfaillie, 2004) and elsewhere (Peuskens, Vanrie, Verfaillie, \& Orban, 2004). In the present article, we present 22 actions, both as movie files from different viewpoints and as 3-D coordinate files. Although as we have mentioned, other action databases are available, we believe that this set can be of use to vision and other researchers. First of all, unlike in the Pollick database, our stimulus set consists of a relatively large number of short, well-delineated actions, all performed by the same actor. Second, in contrast to the pointlight archive of Shipley, the set contains movie files that show the same actions from five different viewing angles. There is indeed evidence that the mental representation of human actions is viewpoint dependent (e.g., Daems \&
Verfaillie, 1999; Olofsson et al., 1997; Verfaillie, 1993, 2000). In addition, we provide the coordinates of the point lights in three dimensions. This has the advantage that it allows researchers to construct customized variations of the point-light stimuli (e.g., depicting actions from other viewpoints than in the movie stimuli we provide).

Third, all actions except one (a tennis serve) can be looped, in the sense that there are no abrupt changes in the location of the dots in the transition from the final frame of the action back to the first one. For most actions, this also follows the natural flow of the movement (e.g., cycling, walking, or rowing); for a few others, this repetition might be less natural (drinking, shooting, playing pool).

\section{Method}

A detailed and extensive discussion of the method has been published previously (Dekeyser et al., 2002), so we limit the description of the stimulus construction to a summary of the major steps in the process (see Figure 1). The movements of a male actor, pantomiming the actions and wearing 30 reflective markers, were recorded by a Qualisys MacReflex motion-capture system (Qualisys, Gothenburg, Sweden), consisting of six $30-\mathrm{Hz}$ position units (i.e., six cameras and corresponding video processors). After the capture session, the 2-D data from all position units were processed off line to calculate the 3-D coordinates of the markers used in the session. Whereas classic motioncapture recordings for point-light stimuli or video recording typically involve 10 to 12 markers, we measured the positions of 30 markers. The 30 markers were placed on anatomical locations specified in Vicon's BodyBuilder 3.5 Manual (Oxford Metrics, 1997). The data from these markers were imported in Character Studio (Autodesk, 1998), a software package that was created for use with 3D Studio MAX (Autodesk, 1997). This allowed
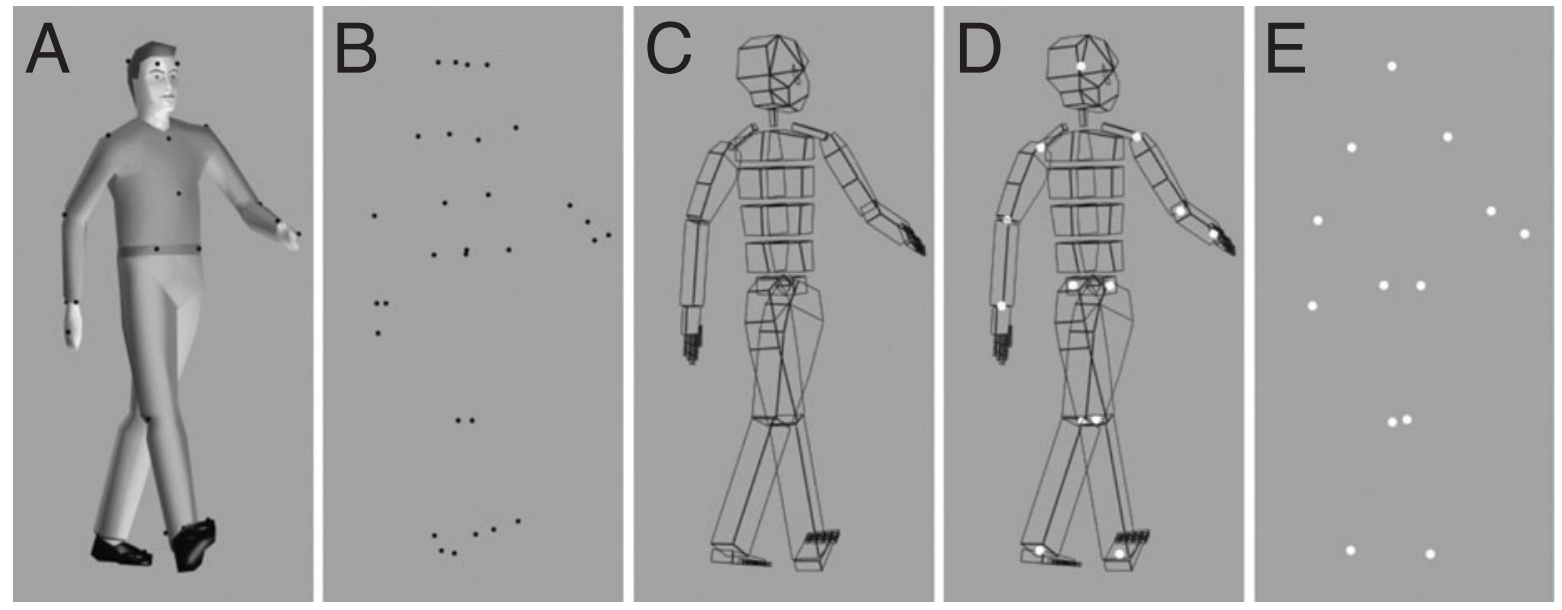

Figure 1. Illustration of the major steps in creating point-light stimuli (figure adapted from Dekeyser, Verfaillie, and Vanrie, 2002). (A) A schematic drawing of an actor with the 30 visible markers attached to the body. (B) The 3-D positions of the markers are registered during a motion-capture session. (C) The captured movement is applied to a biped in Character Studio. (D) Point lights are attached to the major joints of the biped. (E) The resulting point-light stimulus. 
us to animate a biped consisting of a transparent skeleton and 13 bright dots attached to the center of the major joints (head, shoulders, elbows, wrists, hips, knees, and ankles). Next, 3-D coordinates for each of the 13 dots for all frames constituting the action were extracted and some manual smoothing was performed to avoid any remaining "jumpy" dot movements. To create the actual movie files, the smoothed data were imported into 3-D Studio as moving bright spheres and all frames of the action were rendered as .avi files from five different viewpoints. Orthographic projection was used and there was no occlusion, so no explicit depth cues were available. In addition, we also eliminated the global motion of the figure (i.e., the figure does not translate).

Stimuli. The database consists of a single .zip archive that contains a coordinate file (in different file formats) and five movie files for each of the 22 actions; it can be retrieved from the archival website of the Psychonomic Society (www.psychonomic.org) or from the website of the Laboratory of Experimental Psychology of the University of Leuven (http://www.psy.kuleuven.ac.be/labexppsy/actiondb.htm).

Coordinate files. The coordinate files are listings of the 3-D coordinates of the point lights, saved in different file formats: .txt, .xls, and .pdf. The first line in the file provides values for three parameters: the number of frames comprising the action (dependent on the action), the number of markers (always 13), and the frame rate (always $30 \mathrm{~Hz}$ ). The remainder of the file consists of the 3-D coordinates of the 13 markers for each frame of the action. The first 13 lines give the three spatial coordinates of each of the 13 markers in the first frame, in which the first two numbers of a line indicate the values along the horizontal axes and the last number the value along the vertical axis (i.e., the height). The order of the point lights in the file is as follows: head, two shoulders, two elbows, two wrists, two hips, two knees, and two ankles. The next 13 lines give the coordinates for the second frame, and so on.

Movie files. The set of movie files consists of 22 pointlight actions in five different depth orientations: a frontal view $\left(0^{\circ}\right)$, in which the torso is more or less parallel to the projection plane; two lateral views (facing left, $90^{\circ} \mathrm{L}$, and facing right, $90^{\circ} \mathrm{R}$ ); and the two three-quarter views in between (facing left, $45^{\circ} \mathrm{L}$, and facing right, $45^{\circ} \mathrm{R}$ ). The point-light figure, white against a black background, is approximately in the center of the image. Movie files are .avi files with a resolution of 640-480 pixels and a frame rate of 30 frames per second. File sizes are from $60 \mathrm{~K}$ to $200 \mathrm{~K}$. The file name provides both the name of the action and the orientation.

Actions. A short description of each action can be found in Table 1. Clearly, the range of human actions presented here is hardly a representative sample of the whole scope of human movements, but the actions do differ in several respects: First, the action can be symmetrical or not, in the sense that left and right sides of the body perform the same movements (e.g., walking or rowing vs. peddling or playing pool). The symmetrical actions can be either in-phase (e.g., rowing, jumping) or antiphase (e.g., walking, cycling). It should be noted that for walking, the symmetry is perfect; that is, the left side of the body was mirrored to be completely identical to the right side of the body. Second, the action can be instrumental, involving an object or tool, or not (e.g., sawing or peddling vs. crawling or jumping). Third, the action can imply a global motion of the whole body or not (e.g., for walking or rowing, the body normally moves

Table 1

Details of the 22 Actions in the Stimulus Set

\begin{tabular}{|c|c|c|c|c|c|c|}
\hline Action & Description & Symmetrical & Instrumental & $\begin{array}{l}\text { Implied } \\
\text { Global } \\
\text { Motion }\end{array}$ & Loopable & $\begin{array}{c}\text { Length } \\
\text { (frames/msec) }\end{array}$ \\
\hline Chop & two-handed sideways chopping motion & - & + & - & + & $39 / 1,300$ \\
\hline Crawl & moving forward on hands and knees & + (antiphase) & - & + & + & $48 / 1,600$ \\
\hline Cycle & riding a bicycle & + (antiphase) & & + & + & $70 / 2,333$ \\
\hline Drink & bringing glass to mouth and drinking & - & + & - & - & $70 / 2,333$ \\
\hline Drive & steering, turning the wheel & - & + & + & + & $120 / 4,000$ \\
\hline Jump & aerobic-style up-and-down jumping & + (in phase) & - & - & + & $36 / 1,200$ \\
\hline Mow & mowing lawn the old-fashioned way & - & + & - & + & $40 / 1,333$ \\
\hline Paint & a vertical brush stroke & - & + & - & + & $44 / 1,466$ \\
\hline Pedal & pedaling on the front of a boat & - & + & + & + & $42 / 1,400$ \\
\hline Play pool & aiming and shooting at a pool table & - & + & - & - & $122 / 4,066$ \\
\hline Play tennis & amateur tennis serve & - & + & - & - & $101 / 3,366$ \\
\hline Pump & using an old waterpump & - & + & - & + & $51 / 1,700$ \\
\hline Row & rowing a boat & + (in phase) & + & + & + & $65 / 2,166$ \\
\hline Salute & bringing a military salute & - & - & - & - & $95 / 3,166$ \\
\hline Saw & sawing & - & + & - & + & $20 / 666$ \\
\hline Shoot & firing a shotgun & - & + & - & - & $41 / 1,366$ \\
\hline Spade & putting a spade in the ground & - & + & - & + & $49 / 1,633$ \\
\hline Stir & stirring steadily in a bowl & - & + & - & + & $31 / 1,033$ \\
\hline Sweep & sweeping the floor with a broom & - & + & - & + & $37 / 1,233$ \\
\hline Tap & tapping a beer & - & + & - & - & $93 / 3,100$ \\
\hline Walk & perfectly symmetrical walking motion & $+($ antiphase $)$ & - & + & + & $30 / 1,000$ \\
\hline Wave & stepping from side to side with arms up & - & - & - & + & $77 / 2,566$ \\
\hline
\end{tabular}


Table 2

Summary of Spontaneous Naming Responses of 11 Observers

\begin{tabular}{|c|c|c|c|}
\hline Action & $\begin{array}{l}\text { Number of Observers } \\
\text { Who Correctly } \\
\text { Identified the Action }\end{array}$ & $\begin{array}{l}\text { Most Frequently Used Verb } \\
\text { to Describe the Action (Frequency) }\end{array}$ & $\begin{array}{c}\text { Other, Incorrect Actions Reported } \\
\text { by at Least } 2 \text { Observers (Frequency) }\end{array}$ \\
\hline Chop & 2 & Geen idee-no idea (3) & Slaan-to hit (3) \\
\hline Crawl & 11 & Kruipen - to crawl (10) & - \\
\hline Cycle & 11 & Fietsen-to cycle (11) & - \\
\hline Drink & 4 & Drinken - to drink (4) & Schilderen - to paint (2), slaan - to hit (2) \\
\hline Drive & 9 & (Auto)rijden-to drive (7) & Breien - to knit (2) \\
\hline Jump & 11 & Springen-to jump (11) & - \\
\hline Mow & 3 & Maaien - to mow (2) & Slaan—-to hit (4) \\
\hline Paint & 11 & Schilderen - to paint (11) & - \\
\hline Pedal & 3 & Vegen - to sweep (6) & - \\
\hline Play pool & 5 & Biljarten — to play billiards (3) & Slaan-to hit (2) \\
\hline Play tennis & 9 & Tennissen - to play tennis (9) & Werpen - to throw (2) \\
\hline Pump & 1 & Geen idee-no idea (2) & - \\
\hline Row & 10 & Roeien-to row (9) & - \\
\hline Salute & 9 & Salueren-to salute (8) & - \\
\hline Saw & 11 & Zagen-to saw (11) & - \\
\hline Shoot & 0 & Geen idee-no idea (4) & Dansen - to danse (2) \\
\hline Spade & 8 & Spitten-to spade (3) & Vegen-to sweep (3) \\
\hline Stir & 5 & Roeren - to stir (5) & - \\
\hline Sweep & 11 & Vegen - to sweep (11) & - \\
\hline Tap & 1 & Trekken - to pull (2) & Geen idee-no idea (2) \\
\hline Walk & - & - & - \\
\hline Wave & 11 & Dansen-to dance (6) & - \\
\hline
\end{tabular}

across the visual field, whereas for sawing or playing pool it does not). Note that this global motion is only implied in the present stimuli, because we removed the global motion component. Finally, the action can be "loopable"; that is, it may be repeated (e.g., the step cycle in walking or the specific arm motion during sawing) or not (e.g., saluting, which is normally performed only once). As mentioned, all actions - even those that are normally not repeated — can be "visually looped" (except a tennis serve) in the sense that there are no abrupt changes when the action starts anew.

We have collected some preliminary data regarding spontaneous recognition of these actions by asking $11 \mathrm{ob}-$ servers to provide a name for each stimulus. The observers had no prior experience with point-light figures and were given one view of each of the point-light actions (except walking). Table 2 summarizes the results. Half of the actions were highly identifiable, recognized by at least 9 of the 11 observers. For five actions (jumping, painting, sawing, cycling, sweeping), there was $100 \%$ naming agreement; all observers gave the exact same verbal label. The least identifiable movement patterns were chopping, pumping, and tapping, which were recognized by fewer than 3 observers, and shooting, which was never correctly identified. Once the observers were informed what each action represented, however, they could easily discriminate and identify the different actions.

\section{REFERENCES}

Ahlström, V., Blake, R., \& Ahlström, U. (1997). Perception of biological motion. Perception, 26, 1539-1548.

Autodesk, Inc. (1997). Autodesk 3D studio max (Release 2). Sausalito, CA: Author.
AutodesK, Inc. (1998). Character studio max (Release 2). Sausalito, CA: Author.

CutTing, J. E. (1978). A program to generate synthetic walkers as dynamic point-light displays. Behavior Research Methods \& Instrumentation, 10, 91-94.

CutTing, J. E., \& Kozlowski, L. T. (1977). Recognizing friends by their walk: Gait perception without familiarity cues. Bulletin of the Psychonomic Society, 9, 353-356.

Daems, A., \& Verfaillie, K. (1999). Viewpoint-dependent priming effects in the perception of human actions and body postures. Visual Cognition, 6, 665-693.

Dekeyser, M., Verfaillie, K., \& Vanrie, J. (2002). Creating stimuli for the study of biological-motion perception. Behavior Research Methods, Instruments, \& Computers, 34, 375-382.

DitTrich, W. H. (1993). Action categories and the perception of biological motion. Perception, 22, 15-22.

DitTrich, W. H., Troscianko, T., Lea, S., \& Morgan, D. (1996). Perception of emotion from dynamic point-light displays represented in dance. Perception, 25, 727-738.

Druks, J., \& Masterson, J. (2000). An object and action naming battery. London: Psychology Press.

Fiez, J. A., \& Tranel, D. (1997). Standardized stimuli and procedures for investigating the retrieval of lexical and conceptual knowledge for actions. Memory \& Cognition, 25, 543-569.

Johansson, G. (1973). Visual perception of biological motion and a model for its analysis. Perception \& Psychophysics, 14, 201-211.

Olofsson, U., Nyberg, L., \& Nilsson, L.-G. (1997). Priming and recognition of human motion patterns. Visual Cognition, 4, 373-382.

OXFORD METRICS, LTD. (1997). Body builder version 3.5. Oxford, U.K.: Author.

Peuskens, H., Vanrie, J., Verfaillie, K., \& Orban, G. (2004). Human superior temporal sulcus motion region processes actions portrayed in biological motion stimuli. Manuscript submitted for publication.

Vanrie, J., Dekeyser, M., \& Verfaillie, K. (2004). Bistability and biasing effects in the perception of an ambiguous point-light walker. Perception, 33, 547-560.

Verfaillie, K. (1993). Orientation-dependent priming effects in the perception of biological motion. Journal of Experimental Psychology: Human Perception \& Performance, 19, 992-1013. 
VerfaILlie, K. (2000). Visual perception of human locomotion: Priming effects in direction discrimination. Brain \& Cognition, 44, 192-213.

Verfaillie, K., De Troy, A., \& Van Rensbergen, J. (1994). Transsaccadic integration of biological motion. Journal of Experimental Psychology: Learning, Memory, \& Cognition, 20, 649-670.

\section{ARCHIVED MATERIALS}

The following materials and links may be accessed through the Psychonomic Society's Norms, Stimuli, and Data archive, http://www.psychonomic.org/archive/. To access these files or links, search the archive for this article using the journal (Behavior Research Methods, Instruments, \& Computers), the first author's name (Vanrie) and the publication year (2004).
FILE: Vanrie-BRMIC-2004.zip.

DESCRIPTION: The compressed archive file contains for each of the 22 point-light actions: (1) five movie-files (.avi) showing the action from different viewpoints, and (2) a listing of the 3-D coordinates of the point lights in each frame in different file formats (.txt, .xls and .pdf)

AUTHOR's E-MAIL ADDREss: jan.vanrie@psy.kuleuven.ac.be AUTHOR's WeB SITE: http://www.psy.kuleuven.ac.be/labexppsy/actiondb.htm.

(Manuscript received December 31, 2003; revision accepted for publication July 7, 2004.) 